\title{
MiR-146a Ameliorates Hemoglobin-Induced Microglial Inflammatory Response via TLR4/IRAK1/TRAF6 Associated Pathways
}

\author{
Guang-Jie Liu't, Qing-Rong Zhang't, Xuan Gao', Han Wang'2, Tao Tao ${ }^{3}$, Yong-Yue Gao', \\ Yan Zhou ${ }^{1}$, Xiang-Xin Chen ${ }^{1}$, Wei Li $^{1 *}$ and Chun-Hua Hang ${ }^{1 *}$ \\ 'Department of Neurosurgery, Nanjing Drum Tower Hospital, The Affiliated Hospital of Nanjing University Medical School, \\ Nanjing, China, ${ }^{2}$ Department of Neurosurgery, Jinling Hospital, School of Medicine, Southern Medical University \\ (Guangzhou), Nanjing, China, ${ }^{3}$ Department of Neurosurgery, Nanjing Drum Tower Hospital, Clinical College of Nanjing \\ Medical University, Nanjing, China
}

Microglial activation and sustained inflammation in the brain can lead to neuronal damage. Hence, limiting microglial activation and brain inflammation is a good therapeutic strategy for inflammatory-associated central nervous disease. MiR-146a is a promising therapeutic microRNA, since it can negatively regulate the inflammatory response. We thus investigated the expression changes of miR-146a after experimental induction of a subarachnoid hemorrhage (SAH) in vivo and in vitro, and we assessed the anti-inflammatory effects of miR-146a in microglial cells in vitro. Primary microglial cells were preincubated with miR-146a before hemoglobin $(\mathrm{Hb})$ treatment. The results indicated that miR-146a decreased gene expression of $\mathrm{Hb}$-induced pro-inflammatory cytokines (TNF- $\alpha$ and IL-1 $\beta$ ) and phenotype-related genes (iNOS and CD86) through IRAK1/TRAF6/NF-кB or MAPK signaling pathways, suggesting its pro-resolution activity in microglia. However, contrary to the LPS-induced microglia or macrophage activation model, we did not observe an elevation in miR-146a after activation. Overall, our findings demonstrated that miR-146a was involved in the regulation of brain inflammation and could be considered a novel therapeutic agent for treating brain inflammation.

Keywords: subarachnoid hemorrhage, microglial polarization, miR-146a, neuro-inflammation, TRAF6, IRAK1

\section{INTRODUCTION}

Aneurysmal SAH is a devastating disease. EBI is thought to be the main cause of poor outcomes after SAH (Fujii et al., 2013). Inflammation, as a critical role in EBI, has a great impact on the pathology and outcome of $\mathrm{SAH}$, such as blood brain barrier interruption, vasospasm, oxidative stress, or even neuronal death (Miller et al., 2014). Therefore, many efforts have been made to regulate inflammation as a method to avoid excessive damage after SAH.

Abbreviations: Arg1, arginase 1; CD86, cluster of differentiation 86; CD206, cluster of differentiation 206; EBI, early brain injury; GAPDH, glyceraldehyde-3-phosphate dehydrogenase; Hb, hemoglobin; IF, immunofluorescence; IL-1 $\beta$, interleukin 1 beta; IL-4, interleukin 4; IL-10, interleukin 10; iNOS, induced nitric oxide synthases; IRAK1, interleukin-1 receptor-associated kinase 1; LPS, lipopolysaccharide; ROS, reactive oxygen species; RRIDs, research resource identifiers; SAH, subarachnoid hemorrhage; TLR4: Toll-like receptor 4; TNF- $\alpha$, tumor necrosis factor alpha; TRAF6: TNF receptor associated factor 6. 
Microglia are the main glial cells responsible for regulating inflammation. The immune response can be characterized by different cellular phenotypes that are triggered by various factors, and phenotype switching can occur with the progression of disease (Aguzzi et al., 2013; Hu et al., 2015; Lan et al., 2017). Among those phenotypes, pro-inflammatory polarization is characterized by the release of many pro-inflammatory factors (TNF- $\alpha$, IL-1 $\beta$, CD86, and iNOS), while anti-inflammatory polarization present with the expression of many antiinflammatory or phagocytosis-related proteins (IL-4, IL-10, Arg1, and CD206). Our previous studies have demonstrated that inhibiting pro-inflammatory polarization and promoting anti-inflammatory polarization improved neurological function after SAH (Sun et al., 2013; Zhang et al., 2016; Tao et al., 2019). In pro-inflammatory polarization, the TLR4/NF- $\mathrm{B}$ signaling pathway is significantly activated (Hanafy, 2013; Lu et al., 2018), making it the dominant target of drugs for attenuating inflammation (Sheedy et al., 2010; Rahimifard et al., 2017).

MicroRNAs are small ( $\sim 22 \mathrm{nt})$, non-coding RNA molecules. They function at the posttranscriptional stage and bind to the 3' untranslated regions (3' UTRs) of target messenger RNA transcripts (mRNAs), usually leading to gene silencing (Bi et al., 2009; Xie et al., 2013). MiR-146a was found to play an important role in regulating of the inflammatory process. Many cell types under different pathologic conditions are regulated by miR-146a, such as human retinal endothelial cells (Cowan et al., 2014), macrophages (Alam and O'Neill, 2011), and human gingival fibroblasts (Xie et al., 2013). For disease such as Alzheimer's disease, miR-146a was also found to be a potential treatment target (Wang et al., 2012). Among the functional mechanisms of miR-146a, TRAF6 and IRAK1 are two key recognized target genes in the TLR4 signaling pathway (Taganov et al., 2006), i.e., miR-146a functions as a dominant negative regulator of TLR4/IRAK1/TRAF6/NF- $\kappa$ B signal transduction (Ma et al., 2011; Quinn and O’Neill, 2011; Saba et al., 2014).

Thus, we conducted a study to observe the expression pattern of miR-146a and then explored whether the exogenous addition of miR-146a could have an impact on microglial polarization resulting in a neuronal protection.

\section{RESULTS}

\section{MiR-146a Was Reduced in an Experimental Subarachnoid Hemorrhage Model in vivo and in $\mathrm{Hb}$-Stimulated Primary Microglia in vitro}

To determine the endogenous expression pattern of miR-146a after SAH, we assessed levels over a time course in an animal model in vivo and in $\mathrm{Hb}$-activated microglia in vitro. In vivo, miR-146a was evidently reduced at an early stage of $6 \mathrm{~h}$, and the reduction was sustained for 2 days from the onset of experimental $\mathrm{SAH}$ (Figure 1A). It then increased gradually from 3 to 7 days, while the levels were still lower than those of the sham group. A similar expression pattern was observed in primary microglia in vitro (Figure 1B). We detected from 1 to $24 \mathrm{~h}$, that miR-146a was severely attenuated, and then it was subsequently elevated but did not reach the level of the control at $24 \mathrm{~h}$.

\section{MiR-146a Levels Were Greatly Increased After Exogenous Addition}

We transfected miR-146a mimics into microglia in vitro, and after $12 \mathrm{~h}$ we stimulated them with $\mathrm{Hb}$ for $4 \mathrm{~h}$ to determine the transfection effect (Figure 1C). The results showed that with the addition of mimics, the level of miR-146a in microglia was dramatically increased. There was approximately 30-times more miR-146a in the Con+miR-146a (control+miR-146a mimic) group than there was in the Con + nc group (control+miR-146a mimic negative control), and the $\mathrm{Hb}+\mathrm{miR}-146 \mathrm{a}(\mathrm{Hb}+\mathrm{miR}-146 \mathrm{a}$ mimic) group exhibited approximately 30 times more than that of the $\mathrm{Hb}+\mathrm{nc}(\mathrm{Hb}+\mathrm{miR}-146 \mathrm{a}$ mimic negative control) group. This indicated that exogenous addition of miR-146a was successful.

\section{Hemoglobin Induced Strong Pro-Inflammatory Polarization of Primary Microglia}

We next examined mRNA expression of microglial polarization markers (pro-inflammatory markers: TNF- $\alpha$, IL-1 $\beta$, CD86, and iNOS; anti-inflammatroy markers: IL-4, IL-10, Arg1, and CD206) in vitro under $\mathrm{Hb}$ stimulation. For proinflammatory markers, TNF- $\alpha$, IL-1 $\beta$ (cytokines), and CD86 (cell surface marker) showed similar patterns, reaching peaks at $1 \mathrm{~h}$ and then gradually decreasing. iNOS (intracellular marker) levels peaked at $4 \mathrm{~h}$ and subsequently decreased (Figure 2A). For anti-inflammatory markers, IL-10 (cytokines) and CD206 (cell surface marker) were elevated and peaked at $1 \mathrm{~h}$, while IL-4 (cytokines) and Arg1 (intracellular marker) were sustained at significantly reduced levels for $24 \mathrm{~h}$ (Figure 2B).

\section{MiR-146 Attenuated Hemoglobin-Induced Inflammation and Slightly Promoted Anti-Inflammatory Phenotype Switching in the Late Phase of the Time Course}

To investigate the potential anti-inflammatory role of miR-146a on microglia in response to $\mathrm{Hb}$ stimulation, we observed the mRNA expression of the above pro/anti-inflammatory factors at $1,4,12$, and $24 \mathrm{~h}$ (Figure 3). The alterations of TNF- $\alpha$ and IL-1 $\beta$ were the most significant results; they were obviously attenuated at $1 \mathrm{~h}$ (Figure $\mathbf{3 A}$ ), and the reduced levels were maintained until $24 \mathrm{~h}$ (Figure 3D) compared to that of $\mathrm{Hb}+\mathrm{nc}$ group even if their levels were higher than con + nc group (combined with Figure 2). The phenotype markers CD86 and iNOS were reduced at $4 \mathrm{~h}$ (Figure 3B), but tended to increase later. Some anti-inflammatory markers such as IL-4 and CD206 were also inhibited by the addition of miR-146a in 1 or $4 \mathrm{~h}$, and then IL4 gradually increased while CD206 remained reduced. IL-10 was attenuated at $4 \mathrm{~h}$ and did not differ evidently from that of the $\mathrm{Hb}+\mathrm{nc}$ group later. Arg1 was elevated at $12 \mathrm{~h}$ (Figure 3C) and sustained to $24 \mathrm{~h}$ (Figure 3D). These results demonstrated that cellular responses to inflammatory stimuli were very complex. 

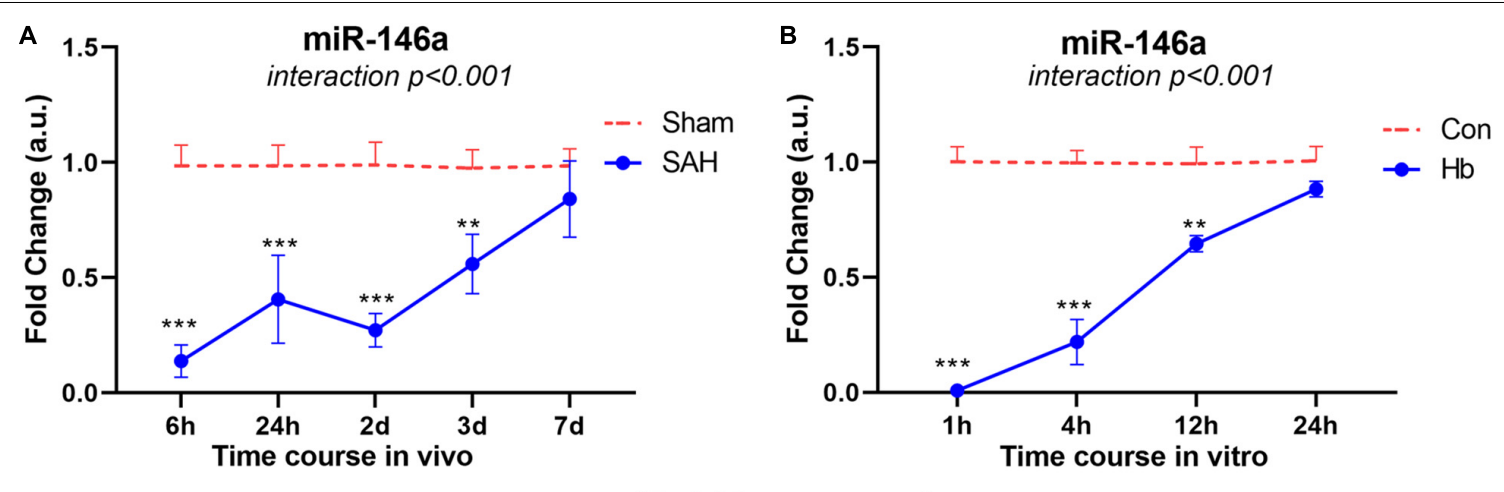

C miR-146a expression

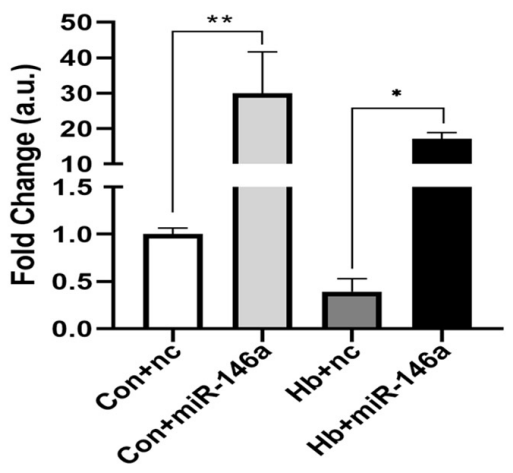

FIGURE 1 | MiR-146a expression after experimental SAH in vivo and in vitro. (A) Time course of miR-146a expression after experimental SAH in vivo from $6 \mathrm{~h}$ to 7 days $(n=3)$. (B) Time course of miR-146a expression after Hb-stimulated microglia in vitro from 1 to $24 \mathrm{~h}(n=3)$. (C) Microglial miR-146a expression change after exogenous addition of its mimics $(n=3)$. Con+nc: control+miR-146a mimic negative control; Con+miR-146a: control+miR-146a mimic; Hb+nc: Hb+miR-146a mimic negative control; Hb+miR-146a: Hb+miR-146a mimic. Data were analyzed by two-way ANOVA (interaction: time*treatment) for A and B; one-way ANOVA followed by post hoc Tukey's test for $\mathbf{C} .{ }^{*} p<0.05,{ }^{* *} p<0.01$, and ${ }^{* * *} p<0.001 . n=$ number of rats or independent cell samples.
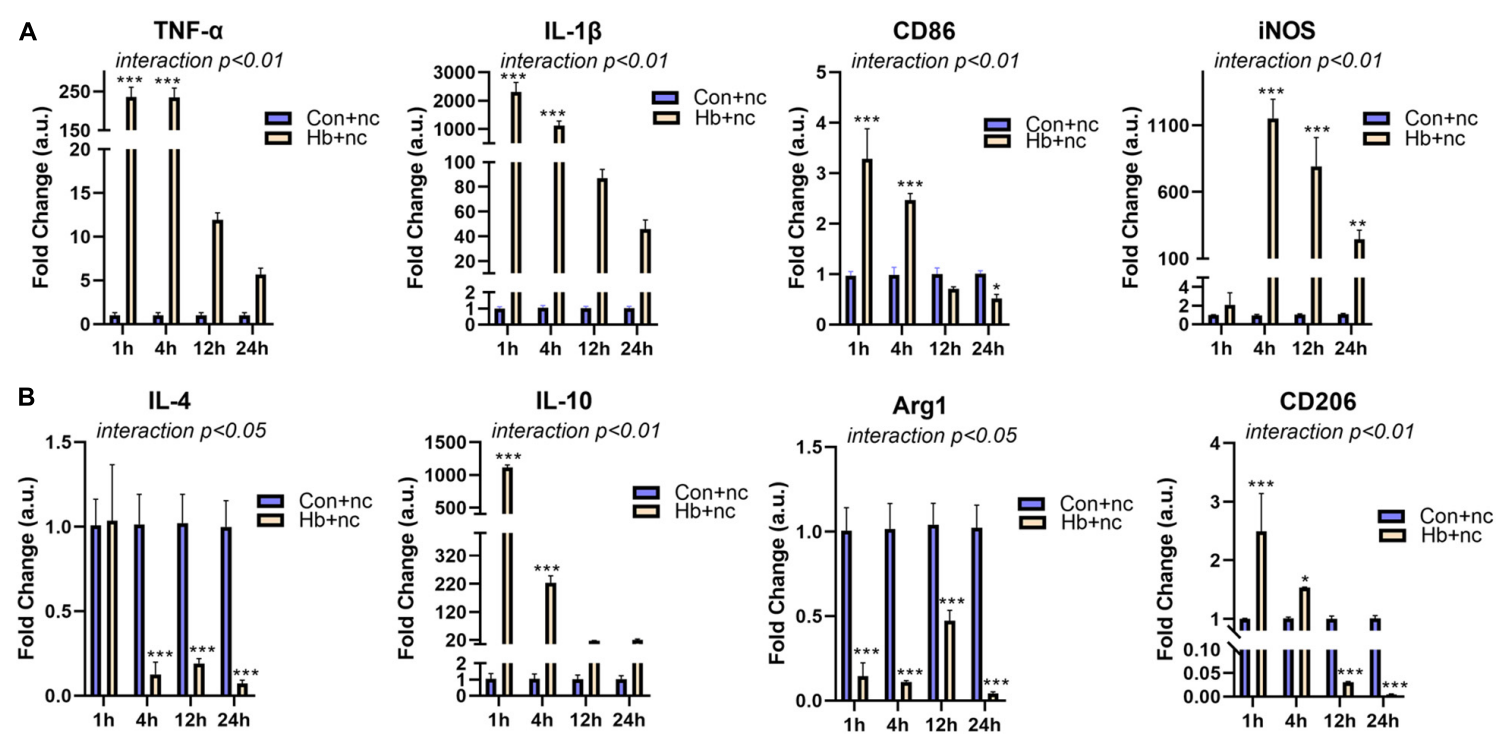

FIGURE 2 | Proinflammatory response and phenotypic modification of Hb-induced primary microglia. Primary microglial cells were treated by $20 \mathrm{\mu M} \mathrm{Hb}$ and a miR-146a negative control (nc, $50 \mathrm{nM}$ ) from 1 to $24 \mathrm{~h}$. (A) mRNA expression of the proinflammatory markers TNF- $\alpha$, IL-1 $\beta$, CD86, and iNOS ( $n=3$ ). (B) mRNA expression of the antiinflammatory markers IL-4, IL-10, Arg1, and CD206 $(n=3)$. Data are presented as the fold change calculated relative to the control+nc group (baseline $=1)$. Values are means $\pm \operatorname{SD}(n=3)$. Data were analyzed by two-way ANOVA (interaction: time*treatment) followed by the Sidak test for multicomparison. ${ }^{*} p<0.05,{ }^{* *} p<0.01$, and ${ }^{* * *} p<0.001 . n=$ independent cell samples. nc miR-146a mimic negative control. 


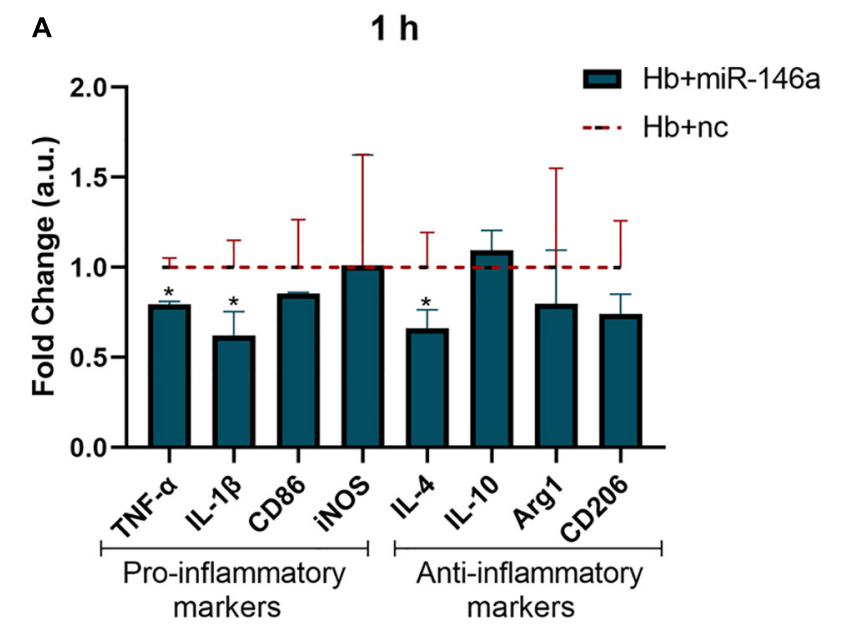

C

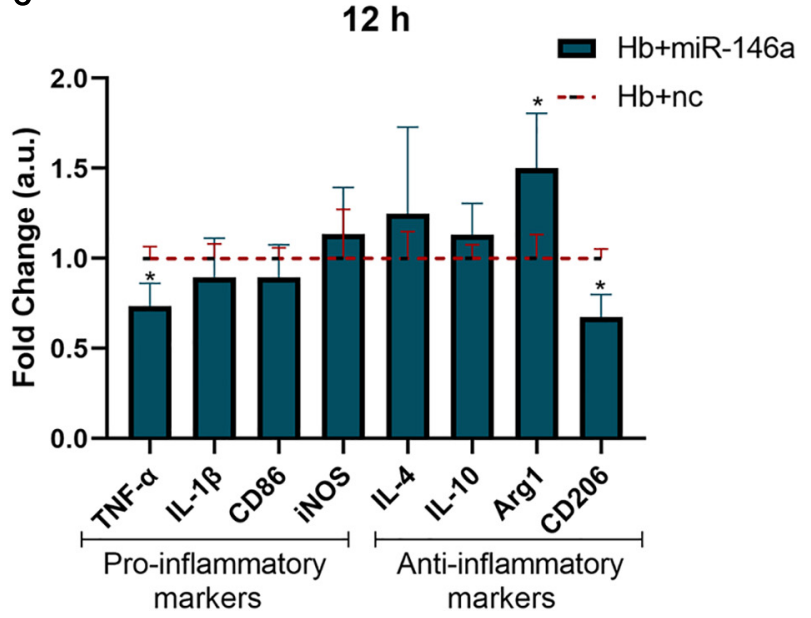

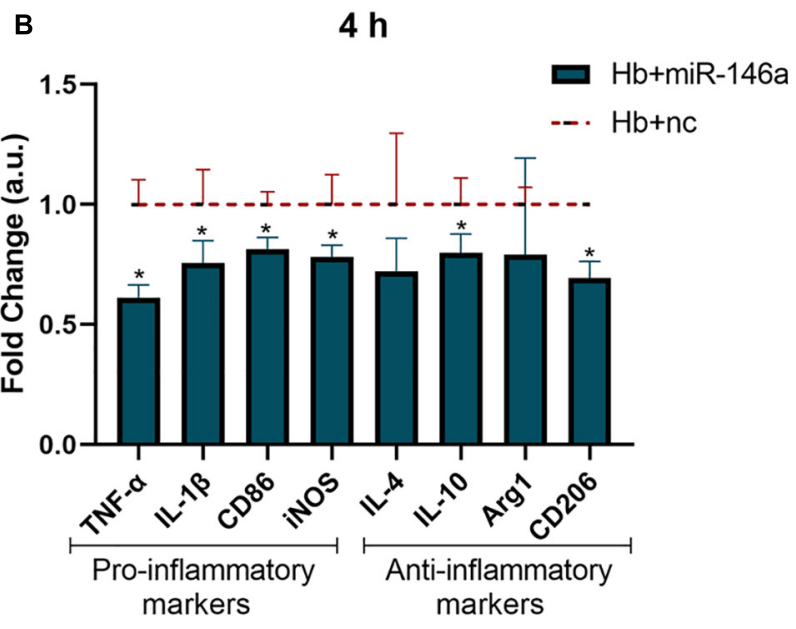

D

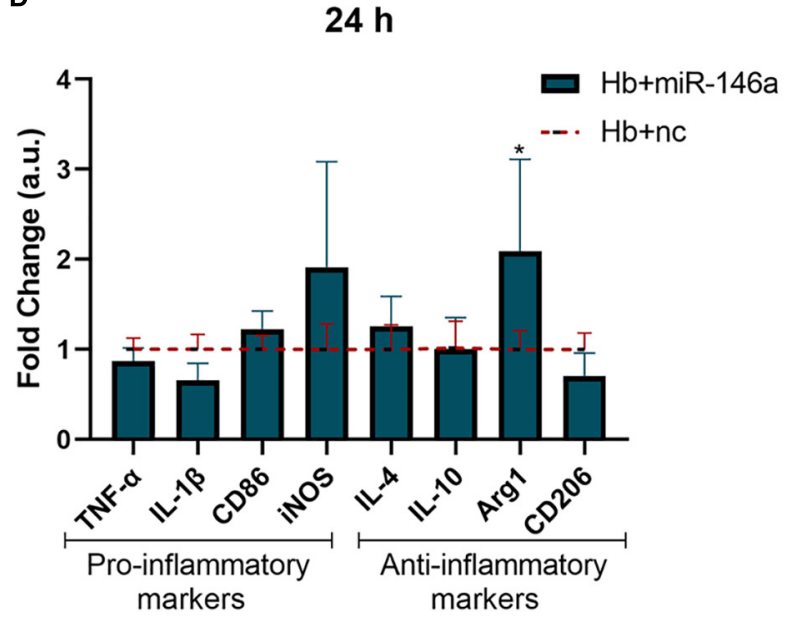

FIGURE 3 | Effect of miR-146a on the $\mathrm{Hb}$-induced inflammatory response and microglial polarization at different time points. Microglia were pretreated with miR-146a $(50 \mathrm{nM})$ for $12 \mathrm{~h}$ and then $\mathrm{Hb}(20 \mu \mathrm{M})$ was added for 1 to $24 \mathrm{~h}$. Data are presented as the fold change calculated relative to the $\mathrm{Hb}+\mathrm{nc}$ group (baseline $=1)$. Values are means $\pm \mathrm{SD}(n=3)$. Statistical analysis was performed using a Mann-Whitney $U$-test for each gene at each time point. ${ }^{\star} p<0.05$. nc miR-146a mimic negative control.

For TNF- $\alpha$ and IL-10, we also examined their protein levels; TNF- $\alpha$ was markedly induced by $\mathrm{Hb}$ and increased from 1 to $24 \mathrm{~h}$ (Figure 4A). MiR-146a reduced its protein levels at 1 and $4 \mathrm{~h}$, not at 12 and $24 \mathrm{~h}$, and even a small elevation was observed at $24 \mathrm{~h}$ (Figure 4C). IL-10 was also increased by Hb but had a reduction at $24 \mathrm{~h}$, while miR-146a had no significant effect on it (Figures 4B,D).

\section{TLR4/IRAK1/TRAF6 mRNA Expression Was Regulated by miR-146a}

We next investigated the influence of miR-146a on the mRNA levels of members of the TLR4/IRAK1/TRAF6 signaling pathway. According to the results, TRAF6 and IRAK1 were markedly reduced by miR-146a and the main time at which a difference in regulation phase was observed was before $12 \mathrm{~h}$ (Figures 5A,B). TLR4 showed a reduction tendency following miR-146a treatment; however, there was no significant difference
(Figure 5C). Then, we detected the effect of miR-146a on P65 translocation to the nucleus (indication of NF- $\kappa \mathrm{B}$ activation) with IF (Figure 5D). Hb induced a rapid and large amount of p65 nuclear translocation, which was decreased by addition of miR-146a (Figures 5D,E).

\section{NF-кB/MAPK Signaling Pathway Activation Was Reduced by miR-146a}

$\mathrm{NF}-\kappa \mathrm{B}$ is a key transcriptional factor triggered by the TLR4 signaling pathway, and it promotes the expression of many proinflammatory cytokines and chemokines in many immune cell types including microglia (Doyle and O'Neill, 2006). To further confirm our hypothesis that miR-146a inhibits TLR4 signaling pathway activation, we detected the downstream proteins involved in TRAF6 and NF- $\kappa$ B activation by Western blotting. Compared to the Con $+n c$ group, TRAF6 protein expression was reduced after the addition of miR-146a, while 

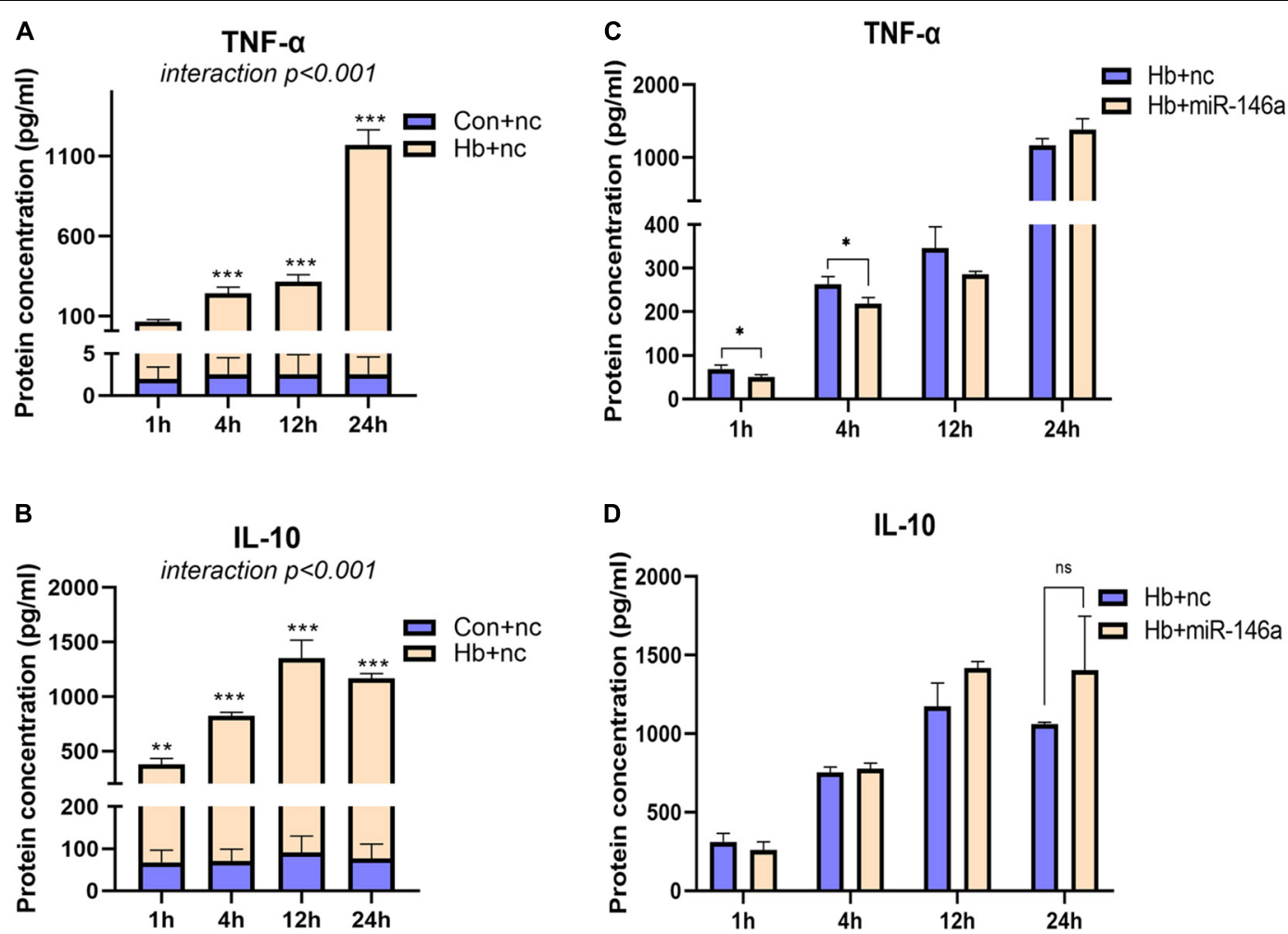

FIGURE 4 | Time-dependent effect of miR-146a on Hb-induced TNF-a and IL-10 release. Cell culture supernatant was collected for examination. (A,B) Primary microglial cells were treated with $20 \mu \mathrm{M} \mathrm{Hb}$ from 1 to $24 \mathrm{~h}$. (C,D) Microglia were pretreated with miR-146a (50 nM) or a miR-146a negative control (nc, 50 nM) for $12 \mathrm{~h}$, and then $\mathrm{Hb}(20 \mu \mathrm{M})$ was added for 1-24 h. Data are presented as the real protein concentration (A,B) or fold change calculated relative to the Hb+nc group $(\mathbf{C}, \mathbf{D}$ baseline $=1)$. Values are means $\pm \mathrm{SD}(n=3)$. Statistical analysis was performed using a two-way ANOVA (interaction: time*treatment) which was followed by the Sidak tests for multicomparison $\mathbf{( A , B )}$ or one-way ANOVA followed by post hoc Tukey's tests for each gene. ${ }^{*} p<0.05$, ${ }^{\star \star} p<0.01$, ${ }^{\star \star \star} p<0.001$. nc miR-146a mimic negative control.

there was no significant change in the phosphorylation of p65 protein (activation of p65). Hb greatly induced phosphorylation of p65 over what was observed in the Con+nc group. MiR-146a decreased the phosphorylation of p65 (Figures 6A,B).

In addition to NF- $\kappa \mathrm{B}$, the MAPK pathway is known to be involved in TLR4-mediated microglial inflammatory responses. We therefore detected the proteins jnk, p44/42 and p38 and their phosphorylation conditions. The three protein levels were markedly elevated after $\mathrm{Hb}$ stimulation, as revealed by comparing the Con+nc group with the $\mathrm{Hb}+$ nc group. MiR-146a reduced the phosphorylation of jnk; however, it had almost no impact on the phosphorylation p44/42 or p38 (Figure 6).

\section{Proinflammatory Microglial Activation-Induced Neuronal Death Was Inhibited by miR-146a}

Neuronal death is a severe pathological process in many acute or chronic diseases of central nervous system. For SAH, inflammation is a critical cause of neuronal death. Thus, we used conditioned medium (CM) from activated microglia to culture primary neurons, enabling us to observe whether miR146a could reduce neuronal damage. $\mathrm{CM}$ from the $\mathrm{Hb}+\mathrm{nc}$ group resulted in significant damage to neurons, characterized by soma shrinkage or neurite degeneration (black arrows) (Figure 7A). ROS production (Figures $\mathbf{7 B}, \mathbf{E}$ ), and $\mathrm{LDH}$ release (Figure $\mathbf{7 C}$ ) were also elevated in the $\mathrm{CM}-\mathrm{Hb}+\mathrm{nc}$ group compared to the levels in the CM-Con+nc group, while cell viability was reduced (Figure 7D). However, in the $\mathrm{CM}-\mathrm{Hb}+\mathrm{miR}-146$ a group, neuronal damage was decreased, ROS production was reduced, LDH release was reduced, and cell viability was elevated.

\section{DISCUSSION}

In this study, we demonstrated for the first time that miR-146a is reduced after SAH in vivo and in vitro. Exogenous addition of miR-146a serves as a potent inhibitor of $\mathrm{Hb}$-induced TNF- $\alpha$ and IL- $1 \beta$ gene expression and a regulator of the microglial phenotype in vitro. Our results deepen our comprehension on the beneficial effects of miR-146a in the central nervous system.

The in vitro experiment could not fully mimic SAH in vivo. MiR-146a fold changes were still low until 2 or 3 days, compared to that of primary microglial result recovered at $24 \mathrm{~h}$. Several reasons might account for this. The in vivo circumstance, composed by various cell types accompanied with numerous inter-cellular interactions, is far too complex for the in vitro experiments to replicate equivalent results. Studies concerning 
A
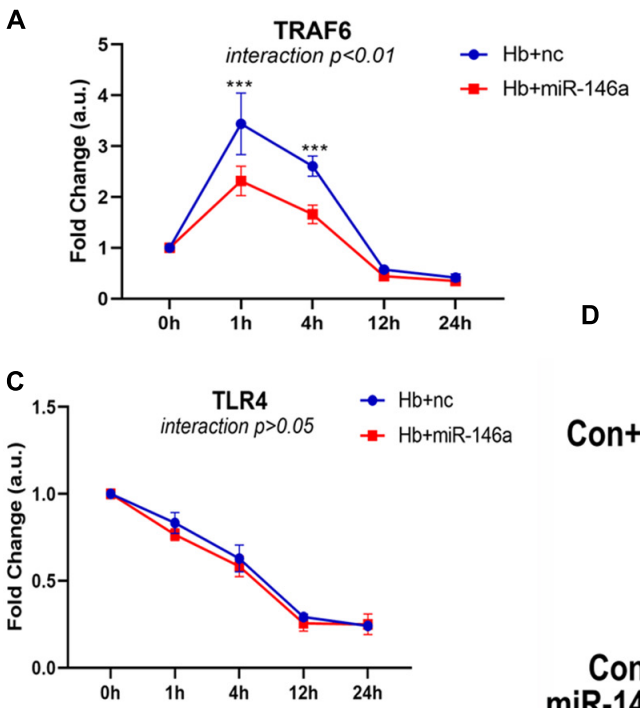

$\mathbf{E}$

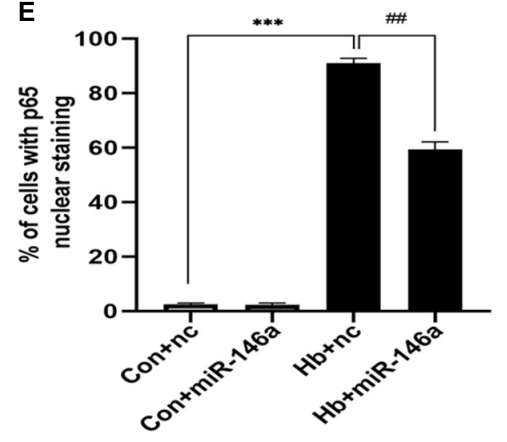

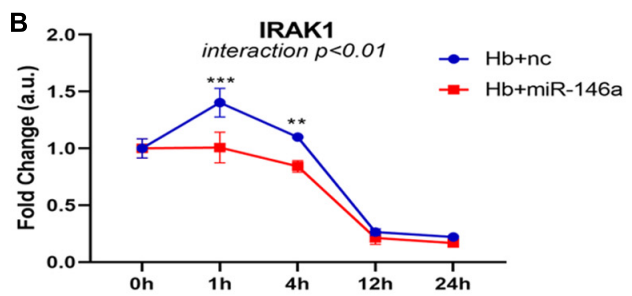

D iba-1 p65 dapi merge
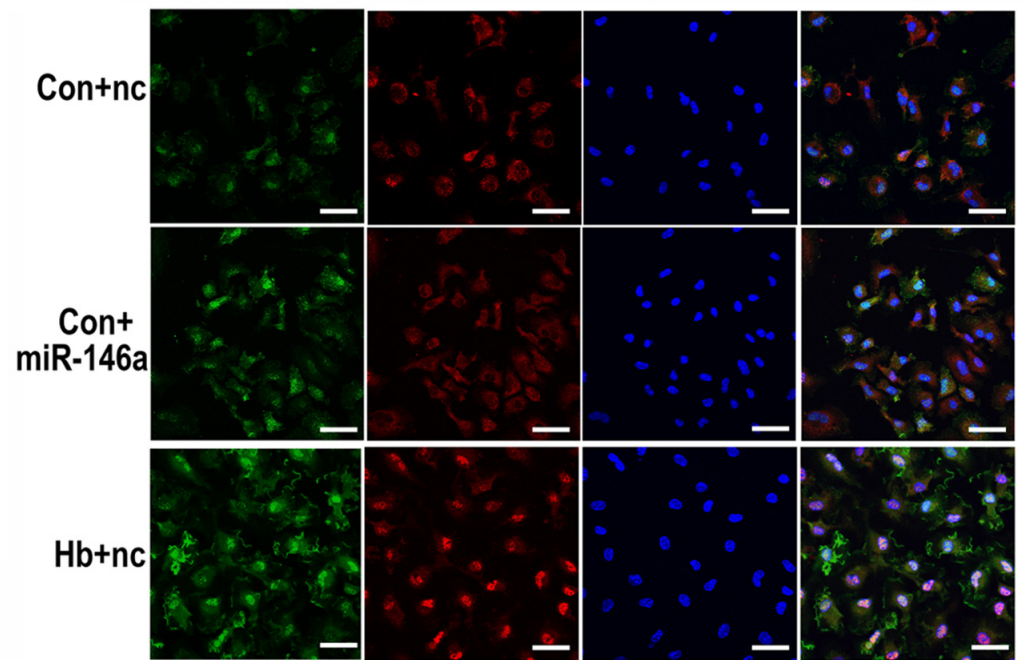

$\mathrm{Hb}+$
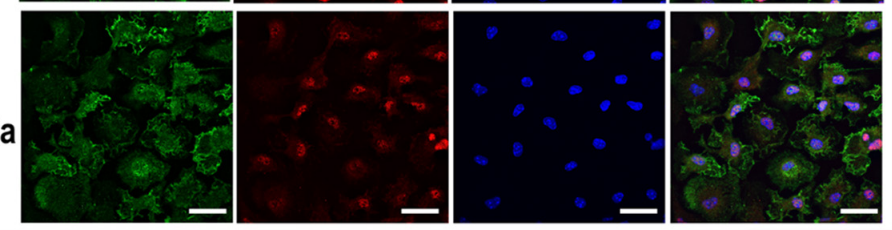

FIGURE 5 | MiR-146a effects on the TLR4/TRAF6/IRAK1/NF-kB signaling pathway. (A-C) Primary microglia were pretreated with miR-146a (50 nM) or miR-146a negative control (nc, $50 \mathrm{nM})$ for $12 \mathrm{~h}$ and then $\mathrm{Hb}(20 \mu \mathrm{M})$ was added for 1-24 $\mathrm{h}$. mRNA expression of TLR4, TRAF6, and IRAK1 was presented as the fold change calculated relative to the con+nc group $(n=3)$. (D,E) Representative images of immunofluorescence of p65 nuclear translocation and corresponding quantification $(n=3)$. Values are means \pm SD. Statistical analysis was performed using a two-way ANOVA (interaction: time*treatment) (A-C) or one-way ANOVA followed by post hoc Tukey's tests for multi-comparison. ${ }^{\star \star} p<0.01,{ }^{* \star \star} p<0.001$, and \#\#p $<0.01$. nc miR-146a mimic negative control. Bar $=40 \mu \mathrm{m}$.

miR-146a in the central nervous system are scarce, while several articles have shined some light on it. Some studies indicated that miR-146a is not just exclusively expressed in microglia, also in astrocytes (Aronica et al., 2010; Iyer et al., 2012; Sison et al., 2017). In addition to that, miR-146a also has impact on neurons (Wang et al., 2014; Jia et al., 2016; Li et al., 2017), oligodendrocytes (Zhang et al., 2017), and endothelial cells (Vasa-Nicotera et al., 2011). Instead of exploring the whole intricate functions of miR$146 \mathrm{a}$, we are particularly interested in how would the miR-146a influence microglial functions.

Subarachnoid hemorrhage is characterized by sterile inflammation, which is different in some ways from LPS-induced inflammation. In our current study, $\mathrm{Hb}$ induced a rapid elevation of pro-inflammatory cytokines, which was similar to the results of LPS stimulation and was consistent with previous in vivo and in vitro studies (Lin et al., 2012; Gram et al., 2013; Kwon et al., 2015). Nevertheless, miR-146a expression was different. Several studies indicated that miR-146a was elevated after LPS stimulation. LPS resulted in rapid elevation of the expression of miR-146a in human monocytic THP-1 cells and BV2 cells (Taganov et al., 2006; Rey et al., 2016). Our study demonstrated that miR-146a was downregulated by $\mathrm{Hb}$ stimulation, indicating a different response due to the differences in cell types or stimulation. In LPS-stimulated THP-1 cells and BV2 cell models, further investigation of miR-146a suggested that the induction is NF- $\kappa$ B dependent. Furthermore, TRAF6 and IRAK1, two critical molecules of the TLR4/NF-kB pathway, are confirmed to be direct targets of miR-146a. This suggests a negative regulatory loop in which NF-kB activation upregulates the miR-146 gene, while the latter downregulates IRAK1 and TRAF6 to reduce the activity of NF-kB (Taganov et al., 2006; Saba et al., 2014). However, although $\mathrm{Hb}$ did not induce elevation of miR-146a in primary microglia, its regulation of the inflammatory response was evident in our results.

Microglial polarization has been shown to be related to cell functions such as motility, phagocytosis, synapse 

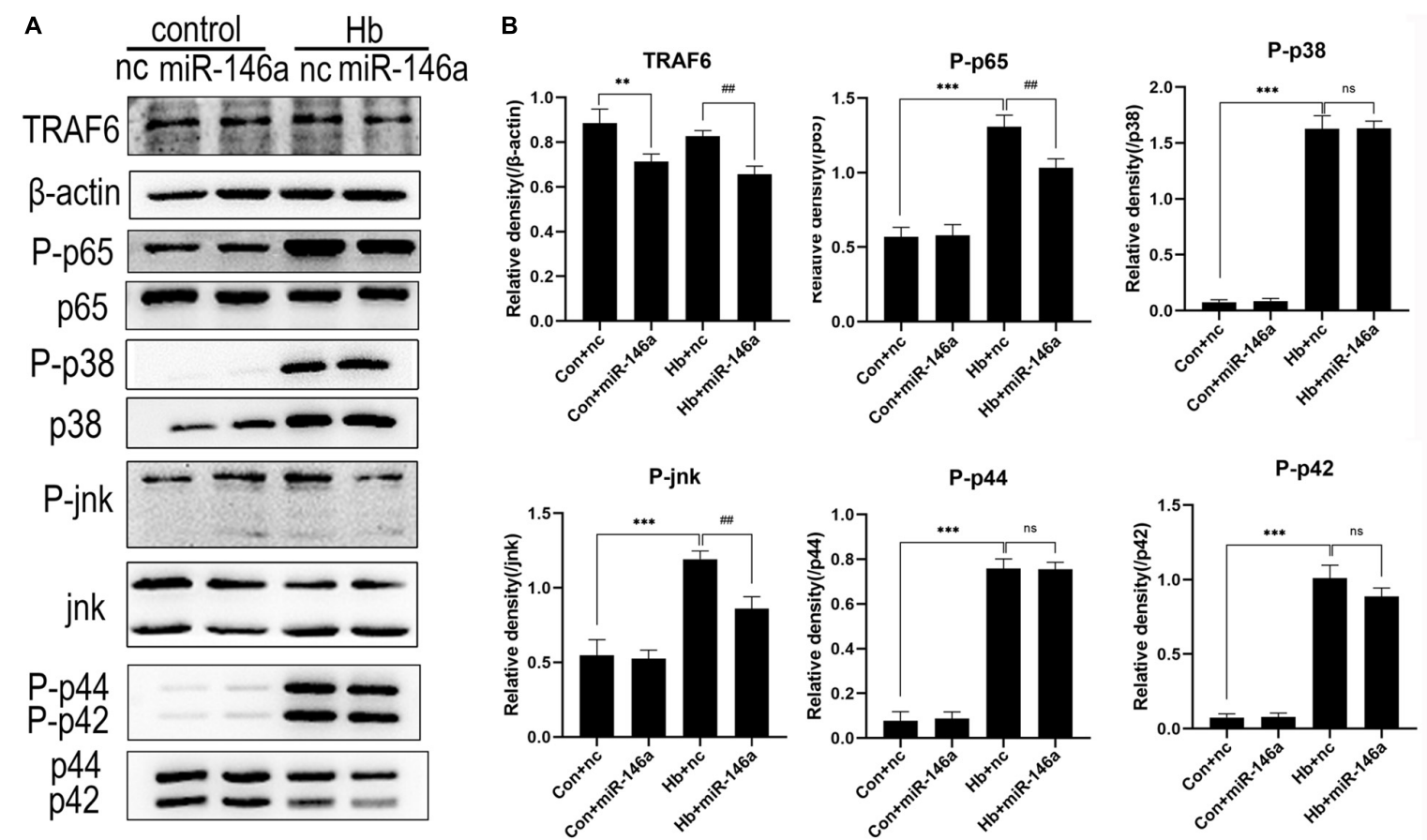

FIGURE 6 | Effects of miR-146a on the expression of proteins in the TLR4/TRAF6/IRAK1/NF-кB signaling pathway. (A) Western blotting results of these proteins. (B) Corresponding quantification analysis of A. Primary microglial cells were preincubated with miR-146a (50 nM) or miR-146a negative control (nc, $50 \mathrm{nM}$ ) for $12 \mathrm{~h}$, and then $\mathrm{Hb}(20 \mu \mathrm{M})$ was added for 15 min. TRAF6 levels were normalized to $\beta$-actin expression, and P-p65, P-p38, P-jnk, P-p44, and P-p42 were normalized to their corresponding non-phosphorylation proteins. Values are means $\pm \mathrm{SD}(n=3)$. Statistical analysis was performed using one-way ANOVA followed by post hoc Tukey's test for multicomparison. ${ }^{\star \star} p<0.01,{ }^{\star \star *} p<0.001$, \#\#p $<0.01$, and ns = no significance. nc miR-146a mimic negative control.

reconstruction, or antigen presentation (Hanisch, 2013). We showed that miR-146a possibly contributed to microglial phenotype modification. In the scenario of pro-inflammatory polarization, TNF- $\alpha$, IL- $1 \beta$, CD86, and iNOS were significantly attenuated at 1 or $4 \mathrm{~h}$ until finally recovered to baseline at 12 or $24 \mathrm{~h}$. For anti-inflammatory polarization markers, IL-4 and IL-10 showed no significant elevation after the addition of miR-146a; contrarily, they were attenuated in the early phase (1 or $4 \mathrm{~h}$ ). Arg1 was not elevated until in the late phase. Most prominently, CD206 was fundamentally inhibited by miR-146a throughout the experimental time course. This receptor is mainly engaged in phagocytosis (East, 2002), and the reason for its continuous attenuation by miR-146a needs further exploration. IL-10 and CD206, elevated after $\mathrm{Hb}$ stimulation, could be inhibited by miR146 a possibly because they were partly regulated by TRAF6. Nevertheless, greater number of samples and more additional polarization markers should be introduced to ensure a more promising explanation. Thus, we conclude that the dominant effect of miR-146a on microglia is pro-inflammatory polarization inhibition, as for the influence on microglial anti-inflammatory polarization, further experiments need to be conducted.

We finally detected the expression of proteins involved in the NF- $\kappa \mathrm{B} / \mathrm{MAPK}$ signaling pathways and of pro-inflammatory cytokines (TNF- $\alpha /$ IL-10). The TRAF6-NF- $\kappa \mathrm{B}$ and JNK were obviously inhibited, but P38 or P44/42 were not. One possible explanation is that some other signaling pathways are engaged in the activation of P38 or P44/42 proteins. TNF- $\alpha$ was significantly attenuated in the early stage ( 1 and $4 \mathrm{~h}$ ) but not at $24 \mathrm{~h}$. This phenomenon was somewhat inconsistent with the change in mRNA expression. Some posttranslational modifications might be involved in this phenomenon. Hence, time of miR-146a treatment should be considered to ensure its good effect in future investigations.

The effect of CM from activated microglia on primary neurons indicated a good protection effect on microglia from intermediate damage. The neurons cultured in CM might suffer from two damage factors: $\mathrm{Hb}$ exposure and inflammatory stimuli. Therefore, it was not clarified whether miR-146a could shield neurons from $\mathrm{Hb}$-induced damage. As our results verified, the inhibition of the inflammatory response could be if not all, part of the reason, while other remaining potential factors also need to be assessed in the future. The results of our in vitro experiments indicated that miR-146a could be used not only for treating SAH also for some other hemorrhage or ischemia-associated diseases in central nervous system, such as cerebral hemorrhage, traumatic brain injury, or ischemic stroke, because TLR pathways are widely activated in the pathological process (Wang et al., 2013; Shi et al., 2019); however, miR-146a related studies in these areas are limited.

Collectively, our preliminary study demonstrated good inhibition by miR-146a of pro-inflammatory polarization of 


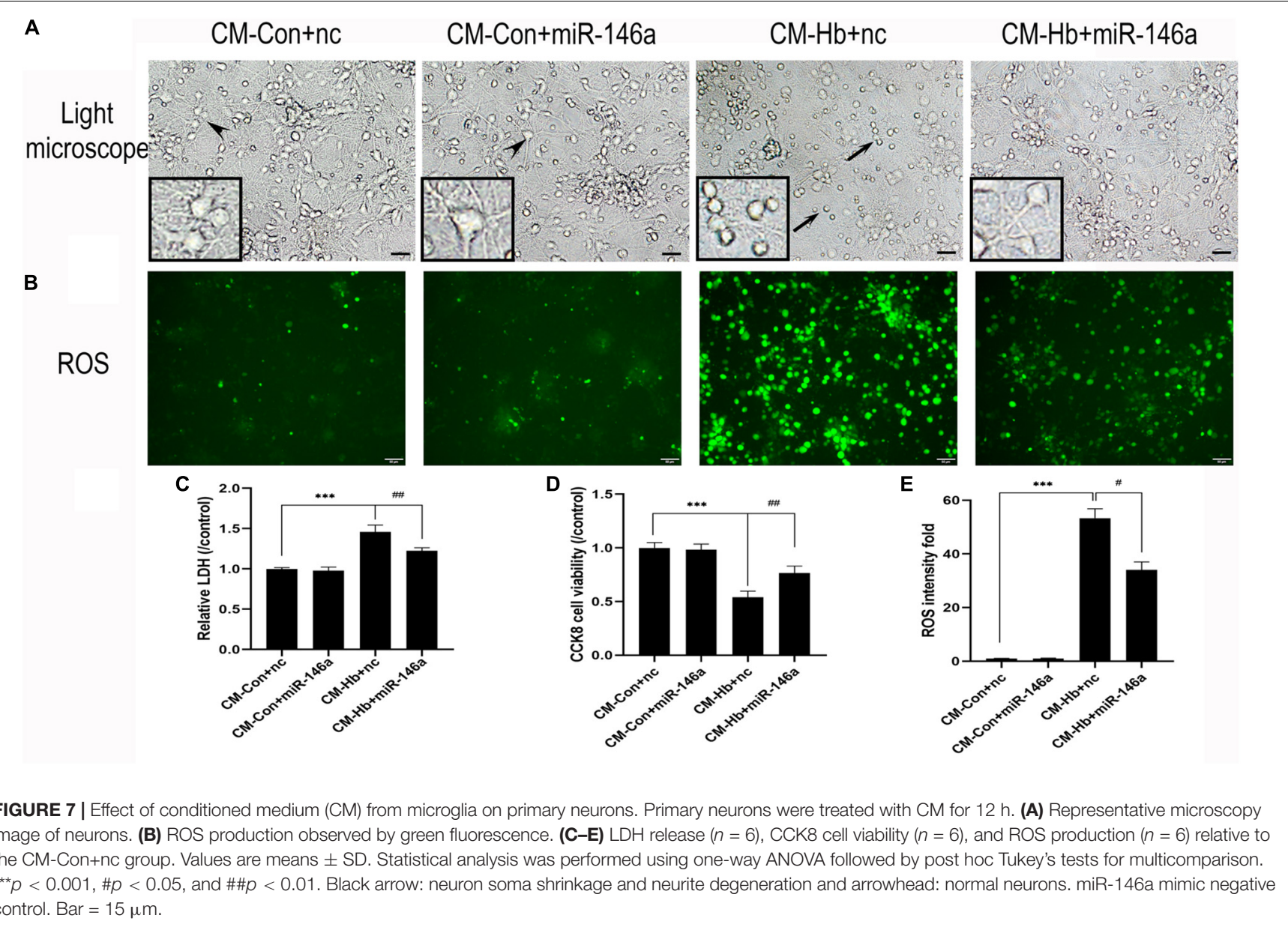

microglia and, to some extent, the promotion of phenotype modification (Figure 8). These effects could attenuate microgliainduced neuron loss and thus exerting neuroprotective effects. However, due to the finite efficacy of in vitro experiment and limited sample size, further attentively designed cellular and animal experiments are needed to get more thorough understanding of the underlying mechanisms.

\section{MATERIALS AND METHODS}

\section{Experimental Protocol}

Experiment 1 was performed in vivo as follows: 30 rats were divided into 10 groups: sham group ( $n=3$ for each time point), SAH 6-h group $(n=3)$, SAH 24 -h group $(n=3)$, SAH 2-day group $(n=3)$, SAH 3 -day group $(n=3)$, and SAH 7-day group $(n=3)$. Total RNA was collected at different time courses, and miR-146a was analyzed.

Experiment 2 was performed in vitro as follows. Primary microglia were cultured for three independent experiments: (a) time course analysis of $\mathrm{Hb}$-induced pro-/anti-inflammatory marker expression between 1 and $24 \mathrm{~h}$; (b) analysis of the effects of miR-146a on the expression of the above markers after different time course treatments with $\mathrm{Hb}$; and (c) identification of the signaling pathway regulated by miR-146a.

Experiment 3 was performed in vitro to determine the effect of CM from microglia on primary neurons. After sufficient treatment, neuron viability were assessed by CCK8 assays $(n=6)$, LDH assays $(n=6)$, and analysis of ROS production $(n=3)$.

\section{Animal Experiments}

32 male Sprague-Dawley rats (RRID: RGD_10395233) weighing 280-320 g were purchased from Animal Core Facility of Nanjing Medical University. Our experiment was approved by the Experimental Animal Ethics Committee of Nanjing Drum Tower Hospital, approved number: 2018020003. Rats were kept in a comfortable environment equipped with a constant temperature of $26 \pm 2^{\circ} \mathrm{C}$ and 12-h light-dark cycles. During the experiment, rats were housed four per cage $\left(57^{*} 36^{*} 14.5 \mathrm{~cm}\right)$ and provided with free access to water and a standard chow diet. Each animal's health condition was estimated every $24 \mathrm{~h}$.

The SAH endovascular perforation model in rats was performed according to previous article (Sehba, 2014). Briefly, rats were transorally intubated and ventilated mechanically during the operation period with $3 \%$ isoflurane anesthesia. A 4-0 monofilament nylon suture was sharpened and inserted into the right internal carotid artery rostrally from the external carotid 


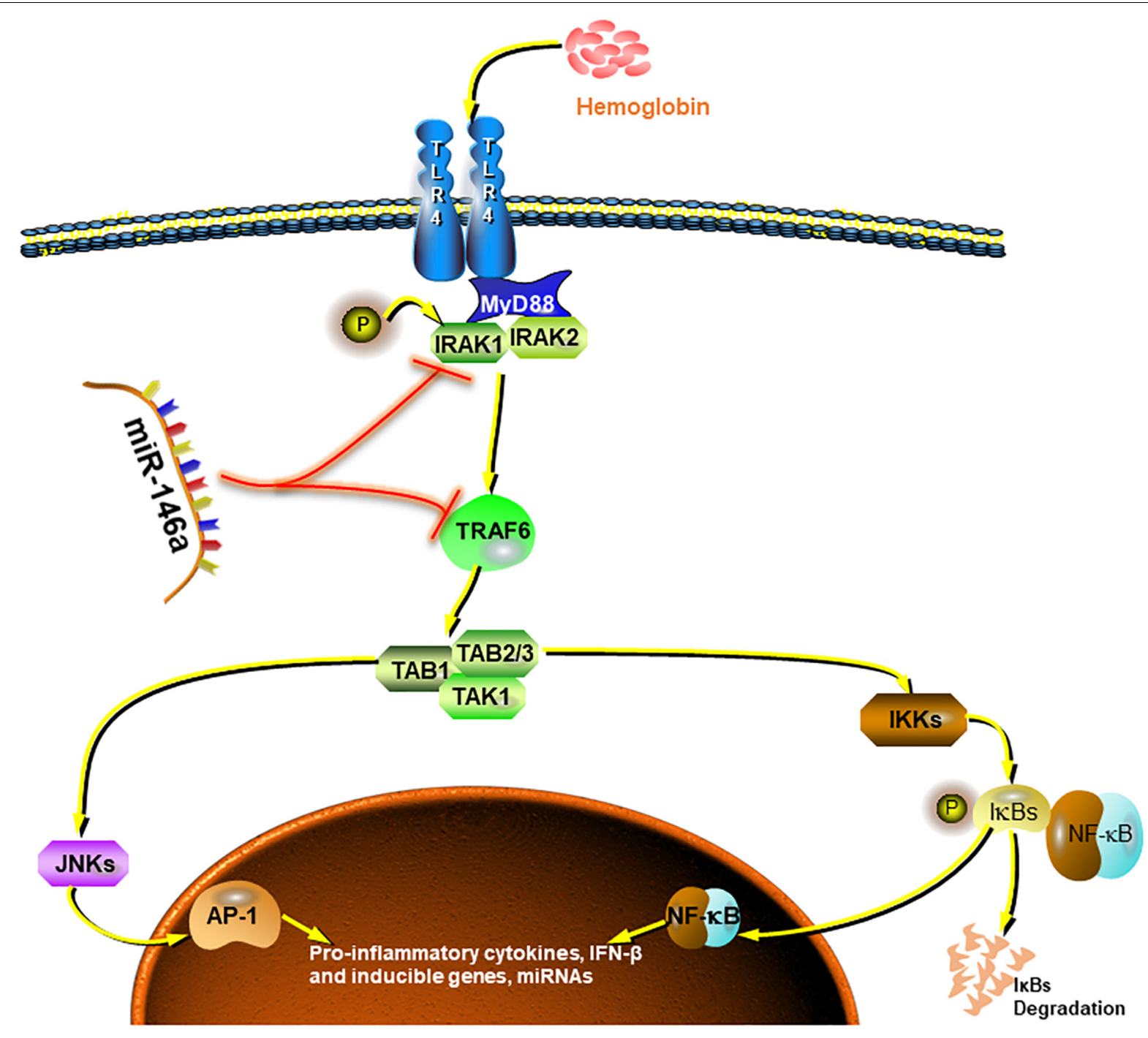

FIGURE 8 | Summarized picture of the effects of miR-146a in this study. MiR-146a attenuates the Hb-induced microglial inflammatory response through TRAF6/IRAK1 inhibition.

artery and punctured the bifurcation of the anterior and middle cerebral arteries. Rats in the sham-operated group underwent the same procedures but the suture was withdrawn without perforated. Aluminum polyester foil blanket was used during and after surgery until rats recovered from anesthesia. Rats were free to water, jelly, or batter of chow diet after surgery.

At each time point, rats were killed by isoflurane anesthesia followed by decapitation and then the brains were removed. The basal cortex tissue was collected and stored at $-80^{\circ} \mathrm{C}$ for further examination.

\section{Primary Microglial Cell Culture and Stimulation}

Primary microglial cells were cultured as described in a previous article (Lian et al., 2016). Briefly, the cerebral cortex was obtained from the brains of neonatal (1 day) mice. After carefully removing the meninges, brain tissue was digested with $0.25 \%$ trypsin (Gibco, United States) for $10 \mathrm{~min}$ at $37^{\circ} \mathrm{C}$. Subsequently, the pellet was triturated with $6 \mathrm{ml}$ of warm culture media with a $1 \mathrm{ml}$ pipet tip. The suspension was filtered through a $70-\mu \mathrm{m}$ strainer (Millipore, Sigma) and centrifuged at $1000 \mathrm{r} / \mathrm{min}$ for $5 \mathrm{~min}$. The remaining cells were resuspended in Dulbecco's modified Eagle's medium (DMEM, Gibco, United States) with the addition of 10\% fetal bovine serum (FBS, biological industries, United States), and then cells were seeded in flasks. Three days after the glial cultures reached confluency, the flasks were shaken for $2 \mathrm{~h}$ at $340 \mathrm{r} / \mathrm{min}$. The floating cells were collected and seeded in plates to obtain microglia.

After seeding on plates for approximately 1-2 days, microglia presented a resting state; then, miR-146a was added to the medium at a concentration of $50 \mathrm{nM}$. After $12 \mathrm{~h}, \mathrm{Hb}(20 \mu \mathrm{M})$ was added and incubated for $1,4,12$, and $24 \mathrm{~h}$. At each time point, cells were collected for further experiments. 


\section{Primary Neuron Culture}

For neuron culture, the cortex was obtained from mice at embryonic day 15 . The next steps were similar to primary microglia culture. After removing the meninges, digestion, titration, and centrifugation were performed, and neurons were seeded on poly-D-lysine precoated plates at a density of $6.0 \times 10^{\wedge} 5 / \mathrm{ml}$ and were incubated at $37^{\circ} \mathrm{C}$ and $5 \% \mathrm{CO} 2$. Four hours later, the medium was completely replaced with neurobasal medium containing 2\% B27 and 1\% GlutMax (Gibco, United States). Neurons were available for use after 7 days of in vitro culture (Ferreira et al., 2014).

\section{MiR-146a Isolation and Quantitative PCR}

Quantitative PCR was performed with a miDETECT A Track ${ }^{\mathrm{TM}}$ system (Ribobio, Guangzhou, China). MiRNA qRT-PCR Primer Sets (one RT primer and a pair of qPCR primers for each set) specific for miR-146a were obtained from this system. All steps were conducted according to the manufacturer's instructions. Total RNA was isolated using TRIzol reagent (Invitrogen, United States). MicroRNAs were reverse transcripted after treatment with a method to achieve poly (A) addition. The qPCR recycling program was $95^{\circ} \mathrm{C}$ for $2 \mathrm{~s}$ and $60^{\circ} \mathrm{C}$ for $30 \mathrm{~s}$, which was performed with SYBER Green mix (Roche, Switzerland) using the a PCR instrument (Applied Biosystems, United States). Each reaction was performed in duplicate and contained a negative control reaction. Data were analyzed by the $2^{-\Delta \Delta \mathrm{Ct}}$ method and U6 was used as an endogenous control.

\section{Western Blotting Analyses}

Cells cultured in six-well plates were washed twice with phosphate-buffered saline (PBS) and lyzed in $100 \mu \mathrm{l}$ of lysis buffer (Thermo Fisher Scientific, MA, United States) per well. Equal amounts of protein extracted from cells were run on a $10 \%$ SDS polyacrylamide gels and then were transferred to a polyvinylidene difluoride membrane (Millipore, Darmstadt, DE, United States). Membranes were blocked for $1 \mathrm{~h}$ with $5 \%$ skim milk and then were incubated at $4^{\circ} \mathrm{C}$ overnight with specific antibodies against phosphorylated or total forms of the following antibodies (all purchased from Cell Signaling Technology, United States): jnk (1:2000, cat. No. 4668S), P-jnk (cat. No. 81E11), p65 (1:1000, cat. No. D14E12), P-p65 (1:1000, cat. No. 93H1), p44/42 (1:1000, cat. No. 137F5), P-p44/42 (1:1000, cat. No. D13.14.4E), p38 (1:1000, cat. No. D13E1), P-38 (1:1000, cat. No. D3F9), TRAF6 (1:2000, 66498-1-Ig, Proteintech, Wuhan, China), and $\beta$-actin (1:2000 cat No. BS6007M, Bioworld Technology, Minneapolis, MN, United States). Membranes were washed three times with Tris-buffered saline containing Tween (TBST) and were subsequently incubated at room temperature for $1 \mathrm{~h}$ with the corresponding horseradish peroxidase (HRP)conjugated IgG (cat No. BS13278 or BS30503, Bioworld Technology, Minneapolis, MN, United States). Protein signals were developed with an enhanced chemiluminescence solution (cat No. 46640, Thermo Fisher Scientific, Waltham, MA, United States). Band gray intensity was quantified with ImageJ software (RRID: SCR_003070).

\section{ELISA}

Primary microglial cells $\left(6 \times 10^{\wedge} 5\right.$ cells/well $)$ that were in 12 -well plates preincubated with miR-146a for $12 \mathrm{~h}$, which was followed by treatment with $\mathrm{Hb}(20 \mu \mathrm{M})$ for $1,4,12$, and $24 \mathrm{~h}$. The culture medium was collected and centrifuged, and the supernatants were stored at $-80^{\circ} \mathrm{C}$ until analysis. The levels of IL-10 and TNF$\alpha$ were determined by ELISA according to the manufacturer's instructions. A standard curve was generated using the mouse IL-10 and TNF- $\alpha$ standards (concentration range $0-1000 \mathrm{pg} / \mathrm{ml}$ ) that were provided in the kits for each assay.

\section{Quantitative PCR (qPCR)}

Primary microglial cells $\left(6 \times 10^{\wedge} 5\right.$ cells/well $)$ were seeded in 12 well plates. The cells were preincubated with miR-146a for $12 \mathrm{~h}$, which was followed by $\mathrm{Hb}(20 \mu \mathrm{M})$ stimulation for $1,4,12$, and $24 \mathrm{~h}$. Total RNA was extracted using TRIzol reagent according to the manufacturer's instructions. The purity and concentration of total RNA were quantified with a BioPhotometer (Eppendorf, Germany). Subsequently, total RNA (1.0 $\mu \mathrm{g})$ was reversetranscribed to generate cDNA using a reverse transcription mix (Vazyme, Nanjing). qPCR was performed using a PCR system (Applied Biosystems, United States) with a SYBER Green mix (Roche, Switzerland). The primers for all genes are shown in Table 1. GAPDH was used as an internal control to normalize the expression of each gene. The relative expression of each gene in every group was calculated using the $2^{-\Delta \Delta \mathrm{Ct}}$ method.

TABLE 1 | Primer pairs used in quantitative PCR.

\begin{tabular}{lll}
\hline Primer name & & Sequence \\
\hline IL-1 $\beta$ & Forward & AAGCCTCGTGCTGTCGGACC \\
TNF- $\alpha$ & Reverse & TGAGGCCCAAGGCCACAGG \\
& Forward & CAAGGGACAAGGCTGCCCCG \\
iNOS & Reverse & GCAGGGGCTCTTGACGGCAG \\
& Forward & CAGCTGGGCTGTACAAACCT \\
CD86 & Reverse & CATGGAAGTGAAGCTTCG \\
& Forward & TCTCCACGGAAACAGCATCT \\
IL-4 & Reverse & CTTACGGAAGCACCCATGAT \\
& Forward & GAGATGGATGTGCCAAACGTC \\
IL-10 & Reverse & CTCTCTGTGGTGTTCTTCGTTG \\
& Forward & TAGAGCTGCGGACTGCCTTC \\
Arg1 & Reverse & AGAAATCGATGACAGCGCCTC \\
& Forward & CAGAAGAATGGAAGAGTCAG \\
CD206 & Reverse & CAGATATGCAGGGAGTCACC \\
& Forward & TCAGACGAAATCCCTGCTACTG \\
IRAK1 & Reverse & AGCCTGACCCCAACTTCTCG \\
& Forward & CCACCCTGGGTATGTGCC \\
TRAF6 & Reverse & GAGGATGTGAACGAGGTCAGC \\
TLR4 & Forward & AAAGCGAGAGATCTTCCCTG \\
GAPDH & Reverse & ACTGGGGACAATCACTAGAGC \\
& Forward & CAAGGGATAAGAACGCTGAGA \\
& Reverse & GCAATGTCTCTGGCAGGTGTA \\
& Forward & TCCCAGCTTAGGTCATCAGGT \\
& Reverse & TACGGGACGAGGAAACACTCTC
\end{tabular}




\section{Immunofluorescence Staining}

Immunofluorescence staining was performed according to our previously published Protocols (Sun et al., 2014; Ye et al., 2018). Briefly, brain sections $(6 \mu \mathrm{m})$ were first incubated with primary antibodies against p65 (1:200, 1:1000, cat. No. D14E12, CST), iba-1 (1:200, RRID: AB_2224402) overnight at $4{ }^{\circ} \mathrm{C}$. The next day, brain sections were incubated with corresponding secondary antibodies Alexa Fluor 488 and/or Alexa Fluor 594 (Jackson ImmunoResearch Incorporation, West Grove, PA, United States). Fluorescence was examined under a ZEISS HB050 inverted microscope system. The fluorescently stained cells were analyzed by Image J software.

\section{Neurotoxicity of Activated Microglia-Conditioned Medium on Neuronal Cells}

Neuronal cells were plated in 96-well plates $\left(2 \times 10^{\wedge} 4\right)$ or 24 well plates $\left(2 \times 10^{\wedge} 5\right)$ until maturation at 7 days. Activated microglia-conditioned media (CM) was prepared as follows: (1) the CM from control microglia cells (Control-CM); (2) CM from control+miR-146a group (CM-Con+miR-146a); (3) $\mathrm{CM}$ from $\mathrm{Hb}(20 \mu \mathrm{M})$ stimulated microglia for $12 \mathrm{~h}$ group $(\mathrm{CM}-\mathrm{Hb})$; and (4) $\mathrm{CM}$ from $\mathrm{Hb}(20 \mu \mathrm{M})+$ miR-146a group $(\mathrm{CM}-\mathrm{Hb}+\mathrm{miR}-146 \mathrm{a})$. Then, the media were transferred to primary neuronal cells. Primary neuronal cell viability and LDH release was assessed after a $12 \mathrm{~h}$ incubation by CCK8 assay (Dojindo Laboratories, Japan) and LDH assay (Thermo Scientific, United States) according to the manufacture instructions after $12 \mathrm{~h}$ incubation, respectively, according to the manufacturer's instructions. Changes in the morphology of primary neuronal cells in various groups were observed using a phase-contrast microscope. ROS production was examined by the addition of $10 \mathrm{mM}$ 2,7-dichlorodihydrofluorescein diacetate (DCFH-DA) (Millipore, Sigma) and was observed with green fluorescence under the same exposure times.

\section{Statistical Analysis}

GraphPad Prism (RRID: SCR_002798) for Windows version 7.03 was used to perform statistical analysis. Two-tailed, unpaired Student's $t$-tests were used to compare two experimental groups. One-way ANOVA followed by post hoc Tukey's tests

\section{REFERENCES}

Aguzzi, A., Barres, B. A., and Bennett, M. L. (2013). Microglia: scapegoat, saboteur, or something else? Science 339, 156-161. doi: 10.1126/science.1227901

Alam, M. M., and O'Neill, L. A. (2011). MicroRNAs and the resolution phase of inflammation in macrophages. Eur. J. Immunol. 41, 2482-2485. doi: 10.1002/ eji.201141740

Aronica, E., Fluiter, K., Iyer, A., Zurolo, E., Vreijling, J., van Vliet, E. A., et al. (2010). Expression pattern of miR-146a, an inflammation-associated microRNA, in experimental and human temporal lobe epilepsy. Eur. J. Neurosci. 31, 11001107. doi: 10.1111/j.1460-9568.2010.07122.x

Bi, Y., Liu, G., and Yang, R. (2009). MicroRNAs: novel regulators during the immune response. J. Cell. Physiol. 218, 467-472. doi: 10.1002/jcp. 21639

Cowan, C., Muraleedharan, C. K., O’Donnell, J. J., 3rd, Singh, P. K., Lum, H., Kumar, A., et al., (2014). MicroRNA-146 inhibits thrombin-induced NF-кB were performed to determine the difference between each experimental group when comparing three or more groups. Twoway ANOVA was performed to determine the interaction effect of treatments and time courses. $P<0.05, p<0.01$, and $p<0.001$ are considered statistically significant, showed as * or \#, ns: not significant. No assessment of normality of data was carried out. Data of all experiments were included. And therefore, no outlier tests were performed in this study. No statistical methods were applied to predetermine the sample size. Data are expressed as the means \pm SD.

\section{DATA AVAILABILITY STATEMENT}

All datasets generated for this study are included in the article/supplementary material.

\section{ETHICS STATEMENT}

The animal study was reviewed and approved by the Experimental Animal Ethics Committee of Nanjing Drum Tower Hospital, approved number: 2018020003.

\section{AUTHOR CONTRIBUTIONS}

G-JL, XG, and HW performed the experiments. C-HH, WL, and Q-RZ contributed to the conception and analysis of the experiments. G-JL, Y-YG, TT, YZ, and X-XC were contributors to writing and editing the manuscript. All authors have read and agreed to the published version of the manuscript.

\section{FUNDING}

This work was supported by grants from The National Natural Science Foundation of China (NSFC): Nos. 81870922 (WL), and 81771291 (C-HH), and 81801166 (L-YW); Medical Science and technology development Foundation, Nanjing Department of Health: No. JQX18001 (WL); and the Fundamental Research Funds for the Central Universities No. 021414380361 (WL).

activation and subsequent inflammatory responses in human retinal endothelial cells. Invest. Ophthalmol. Vis. Sci. 55, 4944-4951. doi: 10.1167/iovs.13-13631

Doyle, S. L., and O'Neill, L. A. (2006). Toll-like receptors: from the discovery of NFkappaB to new insights into transcriptional regulations in innate immunity. Biochem. Pharmacol. 72, 1102-1113. doi: 10.1016/j.bcp.2006.07.010

East, L. (2002). The mannose receptor family. Biochim. Biophys. Acta 1572, 364-386.

Ferreira, T. A., Blackman, A. V., Oyrer, J., Jayabal, S., Chung, A. J., Watt, A. J., et al. (2014). Neuronal morphometry directly from bitmap images. Nat. Methods 11 , 982-984. doi: 10.1038/nmeth.3125

Fujii, M., Yan, J., Rolland, W. B., Soejima, Y., Caner, B., and Zhang, J. H. (2013). Early brain injury, an evolving frontier in subarachnoid hemorrhage research. Transl. Stroke Res. 4, 432-446. doi: 10.1007/s12975-0130257-2

Gram, M., Sveinsdottir, S., Ruscher, K., Hansson, S. R., Cinthio, M., Akerstrom, B., et al. (2013). Hemoglobin induces inflammation after preterm intraventricular 
hemorrhage by methemoglobin formation. J. Neuroinflamm. 10:100. doi: 10 . 1186/1742-2094-10-100

Hanafy, K. A. (2013). The role of microglia and the TLR4 pathway in neuronal apoptosis and vasospasm after subarachnoid hemorrhage. J. Neuroinflamm. 10:83. doi: $10.1186 / 1742-2094-10-83$

Hanisch, U. K. (2013). Functional diversity of microglia - how heterogeneous are they to begin with? Front. Cell. Neurosci. 7:65. doi: 10.3389/fncel.2013.00065

Hu, X., Leak, R. K., Shi, Y., Suenaga, J., Gao, Y., Zheng, P., et al. (2015). Microglial and macrophage polarization-new prospects for brain repair. Nat. Rev. Neurol. 11, 56-64. doi: 10.1038/nrneurol.2014.207

Iyer, A., Zurolo, E., Prabowo, A., Fluiter, K., Spliet, W. G., van Rijen, P. C., et al. (2012). MicroRNA-146a: a key regulator of astrocyte-mediated inflammatory response. PLoS One 7:e44789. doi: 10.1371/journal.pone.0044789

Jia, L., Wang, L., Chopp, M., Zhang, Y., Szalad, A., and Zhang, Z. G. (2016). MicroRNA 146a locally mediates distal axonal growth of dorsal root ganglia neurons under high glucose and sildenafil conditions. Neuroscience 329, 43-53. doi: 10.1016/j.neuroscience.2016.05.005

Kwon, M. S., Woo, S. K., Kurland, D. B., Yoon, S. H., Palmer, A. F., Banerjee, U., et al. (2015). Methemoglobin is an endogenous toll-like receptor 4 ligandrelevance to subarachnoid hemorrhage. Int. J. Mol. Sci. 16, 5028-5046. doi: 10.3390/ijms 16035028

Lan, X., Han, X., Li, Q., Yang, Q. W., and Wang, J. (2017). Modulators of microglial activation and polarization after intracerebral haemorrhage. Nat. Rev. Neurol. 13, 420-433. doi: 10.1038/nrneurol.2017.69

Li, S. H., Chen, L., Pang, X. M., Su, S. Y., Zhou, X., Chen, C. Y., et al. (2017). Decreased miR-146a expression in acute ischemic stroke directly targets the Fbxl10 mRNA and is involved in modulating apoptosis. Neurochem. Int. 107, 156-167. doi: 10.1016/j.neuint.2017.01.011

Lian, H., Roy, E., and Zheng, H. (2016). Protocol for primary microglial culture preparation. Bio Protoc. 6:e1989. doi: 10.21769/BioProtoc.1989

Lin, S., Yin, Q., Zhong, Q., Lv, F. L., Zhou, Y., Li, J. Q., et al. (2012). Heme activates TLR4-mediated inflammatory injury via MyD88/TRIF signaling pathway in intracerebral hemorrhage. J. Neuroinflamm. 9:46. doi: 10.1186/1742-2094-9-46

Lu, Y., Zhang, X. S., Zhang, Z. H., Zhou, X. M., Gao, Y. Y., Liu, G. J., et al. (2018). Peroxiredoxin 2 activates microglia by interacting with Toll-like receptor 4 after subarachnoid hemorrhage. J. Neuroinflamm. 15:87. doi: 10.1186/s12974-018$1118-4$

Ma, X., Becker Buscaglia, L. E., Barker, J. R., and Li, Y. (2011). MicroRNAs in NF-kappaB signaling. J. Mol. Cell. Biol. 3, 159-166. doi: 10.1093/jmcb/mjr007

Miller, B. A., Turan, N., Chau, M., and Pradilla, G. (2014). Inflammation, vasospasm, and brain injury after subarachnoid hemorrhage. Biomed. Res. Int. 2014:384342. doi: $10.1155 / 2014 / 384342$

Quinn, S. R., and O'Neill, L. A. (2011). A trio of microRNAs that control Toll-like receptor signalling. Int. Immunol. 23, 421-425. doi: 10.1093/intimm/dxr034

Rahimifard, M., Maqbool, F., Moeini-Nodeh, S., Niaz, K., Abdollahi, M., Braidy, N., et al. (2017). Targeting the TLR4 signaling pathway by polyphenols: a novel therapeutic strategy for neuroinflammation. Ageing Res. Rev. 36, 11-19. doi: 10.1016/j.arr.2017.02.004

Rey, C., Nadjar, A., Buaud, B., Vaysse, C., Aubert, A., Pallet, V., et al. (2016). Resolvin D1 and E1 promote resolution of inflammation in microglial cells in vitro. Brain Behav. Immun. 55, 249-259. doi: 10.1016/j.bbi.2015.12.013

Saba, R., Sorensen, D. L., and Booth, S. A. (2014). MicroRNA-146a: a dominant, negative regulator of the innate immune response. Front. Immunol. 5:578. doi: 10.3389/fimmu.2014.00578

Sehba, F. A. (2014). Rat endovascular perforation model. Transl. Stroke Res. 5, 660-668. doi: 10.1007/s12975-014-0368-4

Sheedy, F. J., Palsson-McDermott, E., Hennessy, E. J., Martin, C., O’Leary, J. J., Ruan, Q., et al. (2010). Negative regulation of TLR4 via targeting of the proinflammatory tumor suppressor PDCD4 by the microRNA miR-21. Nat. Immunol. 11, 141-147. doi: 10.1038/ni.1828

Shi, H., Hua, X., Kong, D., Stein, D., and Hua, F. (2019). Role of Toll-like receptor mediated signaling in traumatic brain injury. Neuropharmacology 145, 259-267. doi: 10.1016/j.neuropharm.2018.07.022
Sison, S. L., Patitucci, T. N., Seminary, E. R., Villalon, E., Lorson, C. L., and Ebert, A. D. (2017). Astrocyte-produced miR-146a as a mediator of motor neuron loss in spinal muscular atrophy. Hum. Mol. Genet. 26, 3409-3420. doi: $10.1093 / \mathrm{hmg} / \mathrm{ddx} 230$

Sun, Q., Dai, Y., Zhang, X., Hu, Y. C., Zhang, D., Li, W., et al. (2013). Expression and cell distribution of myeloid differentiation primary response protein 88 in the cerebral cortex following experimental subarachnoid hemorrhage in rats: a pilot study. Brain Res. 1520, 134-144. doi: 10.1016/j.brainres.2013.05.010

Sun, Q., Wu, W., Hu, Y. C., Li, H., Zhang, D., Li, S., et al. (2014). Early release of high-mobility group box 1 (HMGB1) from neurons in experimental subarachnoid hemorrhage in vivo and in vitro. J. Neuroinflamm. 11:106. doi: 10.1186/1742-2094-11-106

Taganov, K. D., Boldin, M. P., Chang, K. J., and Baltimore, D. (2006). NFkappaB-dependent induction of microRNA miR-146, an inhibitor targeted to signaling proteins of innate immune responses. Proc. Natl. Acad. Sci. U.S.A. 103, 12481-12486. doi: 10.1073/pnas.0605298103

Tao, T., Liu, G. J., Shi, X., Zhou, Y., Lu, Y., Gao, Y. Y., et al. (2019). DHEA attenuates microglial activation via induction of JMJD3 in experimental subarachnoid haemorrhage. J. Neuroinflamm. 16, 243. doi: 10.1186/s12974-0191641-y

Vasa-Nicotera, M., Chen, H., Tucci, P., Yang, A. L., Saintigny, G., Menghini, R., et al. (2011). miR-146a is modulated in human endothelial cell with aging. Atherosclerosis 217, 326-330. doi: 10.1016/j.atherosclerosis.2011. 03.034

Wang, L., Chopp, M., Szalad, A., Zhang, Y., Wang, X., Zhang, R. L., et al. (2014). The role of miR-146a in dorsal root ganglia neurons of experimental diabetic peripheral neuropathy. Neuroscience 259, 155-163. doi: 10.1016/j.neuroscience. 2013.11.057

Wang, L. L., Huang, Y., Wang, G., and Chen, S. D. (2012). The potential role of microRNA-146 in Alzheimer's disease: biomarker or therapeutic target? Med. Hypotheses 78, 398-401. doi: 10.1016/j.mehy.2011.11.019

Wang, Y., Ge, P., and Zhu, Y. (2013). TLR2 and TLR4 in the brain injury caused by cerebral ischemia and reperfusion. Mediators Inflamm. 2013:124614. doi: $10.1155 / 2013 / 124614$

Xie, Y. F., Shu, R., Jiang, S. Y., Liu, D. L., Ni, J., and Zhang, X. L. (2013). MicroRNA-146 inhibits pro-inflammatory cytokine secretion through IL-1 receptor-associated kinase 1 in human gingival fibroblasts. J. Inflamm. (Lond.) 10:20. doi: 10.1186/1476-9255-10-20

Ye, Z. N., Wu, L. Y., Liu, J. P., Chen, Q., Zhang, X. S., Lu, Y., et al. (2018). Inhibition of leukotriene B4 synthesis protects against early brain injury possibly via reducing the neutrophil-generated inflammatory response and oxidative stress after subarachnoid hemorrhage in rats. Behav. Brain Res. 339, 19-27. doi: 10. 1016/j.bbr.2017.11.011

Zhang, J., Zhang, Z. G., Lu, M., Wang, X., Shang, X., Elias, S. B., et al. (2017). MiR-146a promotes remyelination in a cuprizone model of demyelinating injury. Neuroscience 348, 252-263. doi: 10.1016/j.neuroscience.2017. 02.029

Zhang, X. S., Li, W., Wu, Q., Wu, L. Y., Ye, Z. N., Liu, J. P., et al. (2016). Resveratrol attenuates acute inflammatory injury in experimental subarachnoid hemorrhage in rats via inhibition of TLR4 pathway. Int. J. Mol. Sci. 17:E1331 doi: $10.3390 /$ ijms17081331

Conflict of Interest: The authors declare that the research was conducted in the absence of any commercial or financial relationships that could be construed as a potential conflict of interest.

Copyright $\odot 2020$ Liu, Zhang, Gao, Wang, Tao, Gao, Zhou, Chen, Li and Hang. This is an open-access article distributed under the terms of the Creative Commons Attribution License (CC BY). The use, distribution or reproduction in other forums is permitted, provided the original author(s) and the copyright owner(s) are credited and that the original publication in this journal is cited, in accordance with accepted. academic practice. No use, distribution or reproduction is permitted which does not comply with these terms. 